\title{
BMJ Open Singing for people with aphasia (SPA): results of a pilot feasibility randomised controlled trial of a group singing intervention investigating acceptability and feasibility
}

To cite: Tarrant M, Carter M, Dean SG, et al. Singing for people with aphasia (SPA): results of a pilot feasibility randomised controlled trial of a group singing intervention investigating acceptability and feasibility. BMJ Open 2021;11:e040544. doi:10.1136/ bmjopen-2020-040544

- Prepublication history and additional material for this paper are available online. To view these files, please visit the journal online (http://dx.doi. org/10.1136/bmjopen-2020040544).

Received 15 May 2020 Revised 25 November 2020 Accepted 02 December 2020

Check for updates

(c) Author(s) (or their employer(s)) 2021. Re-use permitted under CC BY-NC. No commercial re-use. See rights and permissions. Published by BMJ.

${ }^{1}$ Institute of Health Research, College of Medicine and Health, University of Exeter, Exeter, UK ${ }^{2}$ Institute of Health and Wellbeing, University of Glasgow, Glasgow, UK ${ }^{3}$ Département d'opérations et systèmes de décision, Université Laval, Québec, Québec, Canada ${ }^{4}$ Department of Psychology, University of Exeter, Exeter, UK

Correspondence to

Dr Mark Tarrant;

m.tarrant@exeter.ac.uk

\section{ABSTRACT}

Objectives Pilot feasibility randomised controlled trial (RCT) for the singing groups for people with aphasia (SPA) intervention to assess: (1) the acceptability and feasibility of participant recruitment, randomisation and allocation concealment; (2) retention rates; (3) variance of continuous outcome measures; (4) outcome measure completion and participant burden; (5) fidelity of intervention delivery; (6) SPA intervention costs; (7) acceptability and feasibility of trial and intervention to participants and others involved. Design A two-group, assessor-blinded, randomised controlled external pilot trial with parallel mixed methods process evaluation and economic evaluation.

Setting Three community-based cohorts in the SouthWest of England.

Participants Eligible participants with post-stroke aphasia were randomised 1:1 to SPA or control. Intervention The manualised SPA intervention was delivered over 10 weekly singing group sessions, led by a music facilitator and assisted by an individual with poststroke aphasia. The intervention was developed using the Information-Motivation-Behavioural skills model of behaviour change and targeted psychosocial outcomes. Control and intervention participants all received an aphasia information resource pack.

Outcome measures Collected at baseline, 3 and 6 months post-randomisation, candidate primary outcomes were measured (well-being, quality of life and social participation) as well as additional clinical outcomes. Feasibility, acceptability and process outcomes included recruitment and retention rates, and measurement burden; and trial experiences were explored in qualitative interviews.

Results 0 87 individuals screened, 42 participants were recruited and 41 randomised $(S P A=20$, control=21); 36 participants $(\mathrm{SPA}=17$, control=19) completed 3-month follow-up, 34 (SPA=18, control=16) completed 6-month follow-up. Recruitment and retention (83\%) were acceptable for a definitive RCT, and participants did not find the study requirements burdensome. High fidelity of the intervention delivery was shown by high attendance rates and facilitator adherence to the manual, and

\section{Strengths and limitations of this study}

- This intervention was co-created with different stakeholders, and is an important step towards improving the health and well-being of people living with post-stroke aphasia.

- This is the first randomised controlled trial (RCT) of a singing intervention for people with post-stroke aphasia designed specifically to address psychosocial needs, and was developed as a pilot in accordance with the Medical Research Council guidelines on developing and evaluating complex interventions.

- A mixed methods approach was used to answer questions on acceptability and feasibility of the group singing intervention.

- Although candidate primary outcomes were identified, further secondary psychosocial outcomes were identified that were not tested in the current RCT.

participants found SPA acceptable. Sample size estimates for a definitive RCT and primary/secondary outcomes were identified.

Conclusions The SPA pilot RCT fulfilled its objectives, and demonstrated that a definitive RCT of the intervention would be both feasible and acceptable.

Trial registration number NCT03076736.

\section{INTRODUCTION}

Each year over 100000 individuals in the UK experience a stroke, and although stroke mortality rates are decreasing, associated disability is still a major health concern. ${ }^{1}$ There are approximately 1.2 million stroke survivors in the UK and around a third of these will experience some degree of aphasia. ${ }^{1}$ Aphasia is a complex language disorder that can impact an individual's speech, reading, writing and comprehension of language. 
Aphasia threatens psychosocial health and reduces social participation. ${ }^{2}{ }^{3}$ Individuals living with aphasia report fewer social contacts and social activities, particularly those outside the home, than non-aphasic older adults $^{45}$ and commonly experience social exclusion. ${ }^{6}$ Individuals with reduced social support following a stroke are more likely to suffer depression and have lower levels of overall well-being. ${ }^{7}$ Support to help people with aphasia reintegrate into family, social and community life is lacking. ${ }^{8-10}$ The current study tested the feasibility and acceptability of a new group-based singing intervention that was designed to address the psychosocial difficulties that people with aphasia report.

Research has most often looked at the potential contribution of music and song for speech rehabilitation among people with aphasia, due to the anatomical overlap in the brain networks that process both music and speech. ${ }^{11}{ }^{12}$ A range of case studies and clinical reports, including mainly people with non-fluent 'motor' aphasia, describe instances of ability to sing over-learnt songs among those with poor speech, and ability to sing words and phrases that cannot voluntarily be spoken. ${ }^{13}$ Some research has shown that group singing can improve the rate of speech and its intelligibility among people with aphasia, ${ }^{14}$ and wider research has shown the therapeutic effects of singing for speech rehabilitation among groups of people with neurological language and speech disorders. ${ }^{15-18}$ However, beyond its potential benefits for voice and speech rehabilitation, singing groups for aphasia may also promote better psychosocial outcomes, which has been much less widely studied.

There is growing evidence that group memberships, in general, are beneficial for individuals with chronic health conditions, including people with aphasia. ${ }^{19}$ Belonging to a group can reduce social isolation, provide an important source of self-esteem, offer a basis for receiving social support, help individuals cope with stigma and increase overall well-being. ${ }^{5}{ }^{20}$ Singing is a group activity wellsuited and accessible to people with aphasia. ${ }^{1621}$ There is evidence that many individuals with aphasia retain the ability to sing, ${ }^{16}$ and enjoy participating in music-making activities. $^{2122}$

Zumbansen and colleagues completed a pilot randomised controlled trial (RCT) on singing groups for aphasia, with speech, language and communication as primary outcomes, but also measuring mood and quality of life. ${ }^{23}$ The trials' small sample size does not allow any inferences to be made about these outcomes, but the authors suggest that the social aspect of the intervention might be important for stimulating communication. Tamplin $e t$ al provided initial qualitative evidence that participating in singing groups may improve mood, confidence, sense of support and motivation among people with aphasia. ${ }^{21}$ Similar outcomes were suggested in a focus group study of people with post-stroke aphasia who participated in a single session of singing, and additionally highlighted the potential of singing groups to encourage the formation of social bonds, or social identity. ${ }^{24}$ However, there is a lack of robust evidence such as RCTs which test the effects of singing for psychosocial well-being among people with aphasia following stroke.

\section{DEVELOPMENT AND OBJECTIVES}

This study followed the developmental stages of the Medical Research Council framework for developing and evaluating complex interventions. ${ }^{25}$ The resulting singing groups for people with aphasia (SPA) intervention is a group-based intervention that combines elements of the Information-Motivation-Behavioural skills model of health behaviour change ${ }^{26}$ alongside recent research into group processes in health contexts. ${ }^{20}$ The development of the intervention is reported in the published protocol and development paper. ${ }^{24} 27$

The current study was focused on examining feasibility and acceptability, with specific objectives to: (1) assess the acceptability and feasibility of recruitment, randomisation and allocation concealment; (2) assess retention rates; (3) estimate variance of continuous outcome measures; (4) assess outcome measure completion and participant burden; (5) assess the fidelity of the intervention delivery; (6) assess SPA intervention costs; (7) assess the acceptability and feasibility of the intervention and trial for participants, group facilitators and stroke survivors with aphasia who served as co-facilitators (hereafter 'singing champions').

\section{METHODS}

Study methods are reported in accordance with 'Consolidated Standards of Reporting Trials' guidelines for reporting pilot trials (online supplemental appendix 1), ${ }^{28}$ and recommended procedures for the reporting of groupbased interventions (online supplemental appendix 2) ${ }^{29}$ and qualitative research were followed where possible (online supplemental appendix 3) ${ }^{30}$ Further details are available in the published protocol. ${ }^{27}$

\section{Trial design}

This was a two-group, blind assessor, randomised controlled external pilot trial with parallel mixed methods process evaluation and economic evaluation. Eligible participants who consented were individually randomised 1:1 to SPA or control.

\section{Patient and public involvement}

Patient and public involvement occurred across the trial, including in its development. The SPA intervention was co-created with stroke survivors with aphasia, music facilitators, speech and language therapists (SLTs), health researchers and psychologists. ${ }^{24}$ Stroke survivors with and without aphasia, and at times family members and carers, formed a user group and advised on the continued development and progression of the trial. A stroke survivor with aphasia joined the trial management group and is 
a co-author. The trial steering committee also included a stroke survivor.

\section{Participants}

Inclusion criteria: (1) diagnosis of aphasia following a stroke (confirmed by general practitioners); (2) willingness to be randomised to either the SPA intervention or control group and attend the intervention venue; (3) conversational English pre-stroke and (4) capacity to consent to participate.

Exclusion criteria: (1) under the age of 18 years old; (2) currently attending an SLT programme; (3) intended to relocate outside of the geographical region; (4) participating in another group intervention study or (5) currently attending an existing singing or music group. There were no exclusion criteria based on stage poststroke or comorbidity.

Participants were recruited through several routes: (1) the South West Peninsula Clinical Research Network (Stroke); (2) through SLTs who provided study information and patient information sheets to potential participants; (3) advertising through local support groups and on relevant websites; (4) via local stroke support networks identified through national organisations (eg, the Stroke Association and Different Strokes) and (5) word of mouth, study flyers, adverts and information sheets placed in community settings and on the host university website.

Qualitative interviews were conducted with some participants, facilitators and singing champions. A target sample matrix was developed to reflect a maximum variation sample of participants based on gender, severity of aphasia, age, time of stroke, previous participation in groups and living situation.

\section{Control arm}

All participants (intervention and control) received a resource pack in aphasia-friendly format, constructed for the purposes of the study, which provided information on living with aphasia and the available local community services.

\section{Intervention arm}

The SPA intervention ${ }^{27}$ consisted of 10 weekly sessions delivered in a community facility across three sites in the South-West of England, with each session lasting $90 \mathrm{~min}$. Sessions comprised approximately $45 \mathrm{~min}$ of group singing, with $45 \mathrm{~min}$ allocated to settling in/warm-up, mid-session break and departure. Three separate venues were used: a church hall, a community centre and a dedicated music venue. All venues were arranged such that there were separate singing and social areas. Chairs were arranged in a semi-circle in the singing area with the facilitator opposite (although their location varied across the programme depending on session content). Social areas contained a large table with chairs surrounding so that there was enough space for all participants to be seated around the table. Each venue had a kitchen area, parking and level entry access. The music venue had a substantial array of instruments and technology for music making, production and editing.

The sessions were facilitated by one of two experienced community music leaders who also provided music accompaniment (keyboard or guitar), and were supported by a 'singing champion'. Both facilitator and champion ran all sessions for their assigned group/s which ranged from six to seven participants. Small auxiliary percussion instruments were available for participants to play, and supported the engagement of participants with limited singing ability. These were chosen as instruments readily available and used widely within the community. Session content was flexible and included a range of songs, mainly popular 'classics', suggested by both facilitator and participants. Each group worked toward a final activity, either the development of a 'playlist' or a performance for family/friends to be delivered in the last session. Carers were welcome to support participants and join in with the singing programme.

The intervention was manualised and detailed (1) essential resources, activities and behaviours of facilitators and participants for delivery in all singing sessions (eg, welcome participants to the group, use inclusive language such as 'we' and 'us' to promote group cohesiveness and social identity, give positive collective and individual feedback) and (2) flexible elements that were optional in sessions (eg, encourage sharing of individual success, encourage shared leadership/responsibility). Facilitators were trained by SLTs and the research team ahead of the trial, with a particular focus on effective communication with people with aphasia. Facilitators were also given the National Institute for Health Research ${ }^{31}$ and Stroke Association $^{32}$ guidelines for communication with people with aphasia. Facilitators completed checklists after each session in order to track fidelity to content of the intervention manual elements. All singing sessions were video recorded to support the process evaluation.

\section{Outcome measures \\ Clinical outcomes}

A research team member collected clinical outcome measures at baseline. Follow-up assessments (3/6 months) were conducted by a blinded assessor, and the carer quality of life measure was completed by the carer.

Clinical outcome measures collected at baseline, 3-month and 6-month follow-up were: well-being (ICEpop CAPability measure for Adults (ICECAP-A) $)^{33}$; Stroke and Aphasia Quality of Life Scale (SAQOL-39) ${ }^{34}$; EuroQol Quality of Life Scale (EQ-5D-5L) ${ }^{35}$ and the modified Reintegration to Normal Living Index (mRNLI ${ }^{36}$ measuring social participation. Clinical outcome measures collected at baseline and 6-month follow-up only were: communication (Communication Outcome after Stroke Scale $(\mathrm{COAST}))^{37}$; aphasia (Very Short Version of the Minnesota Aphasia Test) ${ }^{38}$; carer quality of life (CarerQoL 7 -D) ${ }^{39}$ and use of health and social services (Service Receipt Inventory). ${ }^{40}$ The EQ-5D-5L, ICECAP-A and 
mRNLI questionnaires were converted into a format specifically for participants with aphasia, which involved simplified wording, changes to text format and pictorial representations. Participant responses to the aphasia test were reviewed by CC, an aphasiologist, who determined aphasia type and calculated severity of aphasia (total scores of ' $0-17$ ' $=$ 'mild aphasia', ' $18-34$ ' ='moderate aphasia' and '35-51'='severe aphasia'). ${ }^{3841}$

\section{Feasibility, acceptability and process outcomes}

These outcomes included numbers and details of individuals approached; recruitment and retention rates; completion of weekly session checklists by group facilitators; singing group attendance records; interviews with participants, group facilitators and singing champions; and any adverse events (AEs) reported by participants, facilitators or research staff. Participants' experiences of SPA and the trial were drawn from the qualitative interviews.

\section{Qualitative interviews}

Interviews were completed at a time and location convenient to the participant. Apart from one facilitator interviewed at their place of work, and one facilitator, singing champion and participant interviewed at the research offices, all interviews were completed in participant homes. Interviews varied in length, from approximately $20 \mathrm{~min}$ to 1 hour and followed semi-structured topic guides (online supplemental appendix 4). There were some differences compared with a 'standard' approach to qualitative data collection in order to successfully elicit and interpret responses from people who have difficulty in communicating. ${ }^{42}$ For example, based on a prior knowledge of participants' communication difficulties, adaptations were made where necessary, such as offering a range of possible responses, interpreting participant gestures, more closed questioning and allowing input from carers.

\section{Sample size}

The trial recruitment target was 48 participants (24 intervention and 24 control). This target was based on a recommendation of 30 completed data sets for pilot trials to estimate outcome variance ${ }^{44}$ and assuming $20 \%$ attrition, estimated with a precision of $\pm 13 \%$ with $95 \%$ certainty. One researcher (MC) completed 19 interviews (15 trial participants, 2 facilitators and 2 singing champions). Of the 15 participants ( 8 intervention and 7 control) invited to interview, no-one declined, but one control later withdrew their interview data. Data for eight intervention and six control participants were therefore used, five from group A, five from group B and four from group C. Five carers were also consented to accompany participants during interview and contributed to interview content.

\section{Randomisation and blinding}

Exeter Clinical Trials Unit supported a web-based randomisation service with minimisation stratified by aphasia type (fluent vs non-fluent) and severity (mild/moderate vs severe), gender and site (groups A, B and C). The first $20 \%$ of participants were allocated using simple randomisation, with subsequent participants allocated using the minimisation algorithm. The allocation process retained a stochastic element to promote allocation concealment. Participants were allocated 1:1 to the intervention and control groups.

Participants were recruited (consented) individually and randomised once the target cohort number was reached (target $n=16$ for each site) or until expressions of interest were exhausted. As a result of this some participants had to wait for more than 4 weeks between consent and randomisation. After randomisation neither the participants nor research team were blinded to allocation outcome. Follow-up outcome assessments were completed by trained speech and language therapists who were blinded to outcome allocation. Statistical analyses were performed by a statistician, blinded to outcome allocation, using anonymised group allocation codes.

\section{Data analysis}

Recruitment, randomisation, allocation concealment (number of participants who revealed their allocation to an outcome assessor), outcome measure completion and burden were assessed using descriptive statistics and qualitative interview data (objectives 1 and 4). The numbers of participants completing 3-month and 6-month follow-up outcomes were reported with $95 \%$ CIs (objective 2). Participant (patient and carer) health and quality of life related outcomes were reported descriptively at baseline, 3-month and 6-month follow-up; between group differences at 6-month follow-up were also reported with 95\% CIs, adjusted for baseline score, gender, site, aphasia severity and aphasia type. All analyses used the intention to treat principle, whereby participants were analysed according to their allocated group irrespective of the treatment received. Observed data only were used for the analyses, with no use of methods to address missing data (such as multiple imputation).

A sample size estimate for a definitive trial was calculated for potential candidate primary outcomes using the published minimum clinically important difference for each one and SDs derived either from the study or from referenced data (with $90 \%$ power and $5 \%$ alpha and assuming $20 \%$ attrition and 1:1 allocation; objective 3$).{ }^{44}$

Intervention fidelity was assessed using attendance records and the facilitator checklists (objective 5). Checklists contained essential and flexible elements of the intervention manual and facilitators self-reported whether these were included in the session each week. A sample of these checklists were cross-checked with researcher observations, where one researcher $(\mathrm{AB})$ assessed fidelity by completing the session checklist against the video recording of the session.

A micro-costing approach was adopted to estimate the intervention costs associated with SPA (objective 6). The intervention costs for SPA included training costs, staff time during training and delivery of the intervention, 
travel costs for facilitators and singing champions, course materials (song books, percussion instruments, badges and flip charts), venue costs and refreshments. Health resource use included primary care (GP and nurse), secondary care (hospital admissions and accident and emergency) and social care. Healthcare costs were estimated using unit cost reported in Curtis and Burns. ${ }^{45}$

Interview recordings were transcribed verbatim. Qualitative data were analysed using thematic analysis as outlined by Braun and Clarke ${ }^{46}$ to assess acceptability and feasibility for participants, group facilitators and singing champions (objective 7). The coding framework for qualitative data was developed by one researcher (MC) and refined alongside a second researcher $(\mathrm{SD})$, both experienced qualitative researchers with $\mathrm{SD}$ independent of the data collection. All content was then coded (MC) and a sub-set second-coded (AA), the framework refined further, and principal themes discussed between the researchers to ensure balanced interpretation of the data.

\section{RESULTS}

Objective 1: acceptability and feasibility of recruitment, randomisation and allocation concealment

Recruitment took place between May 2017 and April 2018. Of 87 individuals screened (figure 1), 22 were ineligible. In total, $11 / 36$ (31\%, 95\% CI $16 \%$ to $48 \%)$ male invitees and 11/27 female invitees declined to

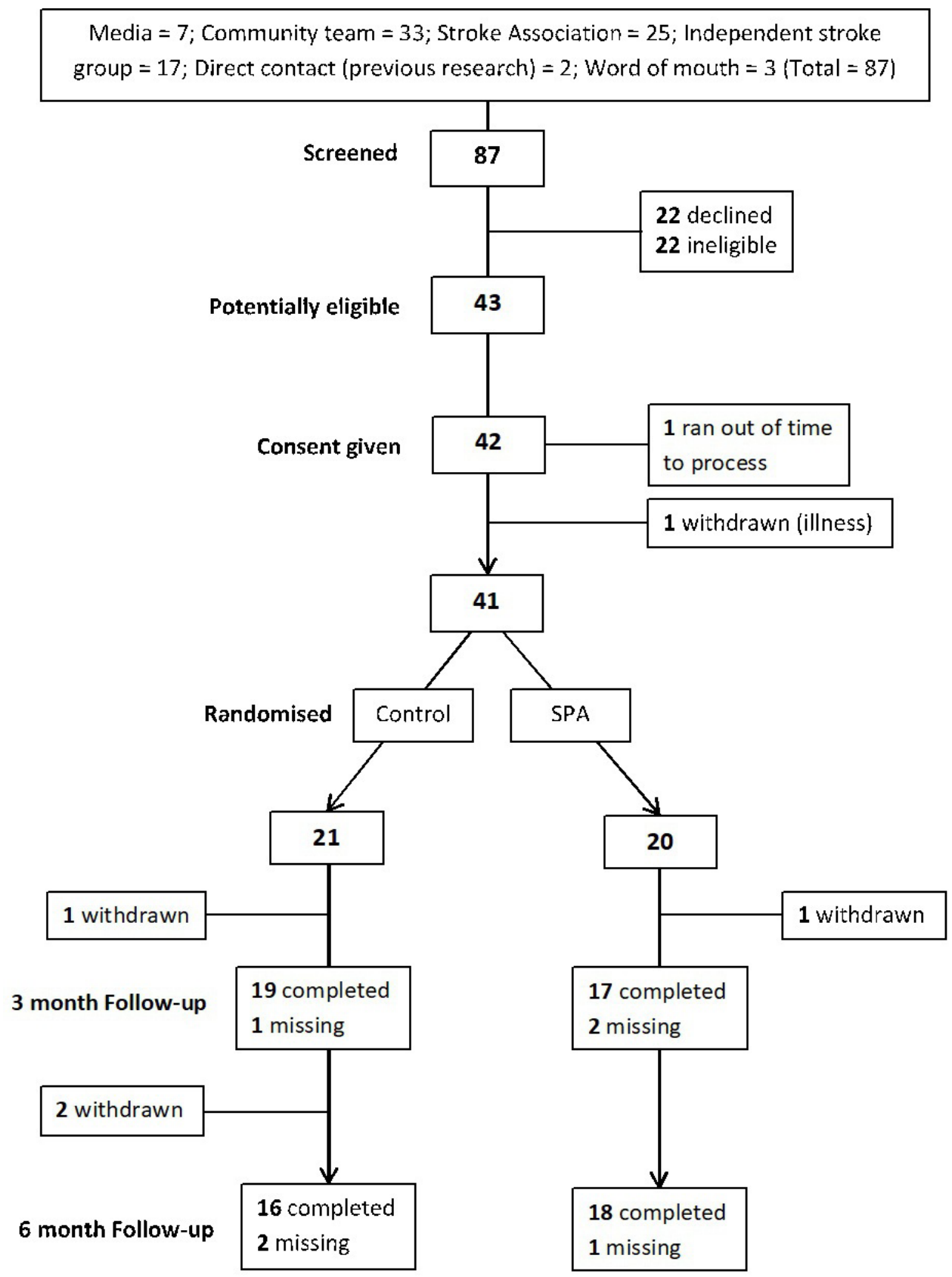

Figure 1 Consolidated Standards of Reporting Trials diagram of participant recruitment and retention. SPA, singing groups for people with aphasia. 
participate $(41 \%, 95 \%$ CI $22 \%$ to $61 \%)$. Of the 42 participants recruited and consented (figure 1), one withdrew prior to randomisation due to ill health, leaving 41 participants randomised: 20 to the intervention and 21 to the control group. The research team, including an aphasiologist (CC) worked to assess capacity to consent. Characteristics of participants at baseline are reported by allocation group (table 1). The SPA group included a higher proportion of participants with another medical problem at baseline compared with the control group $(80 \%$ vs $62 \%)$. A greater proportion of participants in the control were retired compared with the SPA group $(90 \%$ vs $75 \%)$. A greater proportion of participants in the SPA group had received SLT compared with control (85\% vs $67 \%)$.

Across the follow-up assessments, only two individuals revealed their allocation to outcome assessors; one in the control arm (3-month follow-up) and one in the intervention arm (6-month follow-up). For the former participant, a different assessor was used at the 6-month follow-up assessment to minimise bias.

Interview data indicated that participants were generally accepting of the randomisation process. Several intervention participants noted that they would have been disappointed if the randomisation result had been different for them, while some control participants expressed relief that they had not been assigned to the intervention group:

I'm glad I was in the singing group ... than just in the study, 'cos I'm not very good at motivation, just reading the paperwork. (participant)

But when it came through no [control], he was thinking whoopee! (carer)

\section{Objective 2: participant retention rates}

Four participants formally withdrew from the trial postrandomisation (declining to complete follow-up measures), two prior to 3-month follow-up and two subsequently. At 3-month follow-up, 36/39 participants completed the assessments $(88 \%$ of the total 41 randomised participants, $95 \%$ CI $74 \%$ to $96 \%)$; at 6 -month follow-up, $34 / 37$ completed the assessments $(83 \%$ of the total 41 randomised participants, $95 \%$ CI $68 \%$ to $93 \%$ ). In summary, the retention rate was below $20 \%$, comparable to that seen in other similar trials. ${ }^{47}$ Although the target recruitment of 48 participants was not met, the overall low levels of attrition meant that data were collected from a sufficient number of participants to meet objective 3 .

\section{Objective 3: variance of continuous outcome measures}

Participant outcome data (table 2) were collected at baseline ( $\mathrm{n}=41), 3$ months $(\mathrm{n}=36)$ and 6 months $(\mathrm{n}=34)$. Means and SDs for all outcomes are reported at baseline and at follow-up. The SD derived from this participant group can be used to inform power calculations for the target primary outcomes in a definitive trial (see table 3 ).
Objective 4: outcome measure completion and participant burden

There was a high level of completion (at least $85 \%$ ) of outcome measures at each assessment point, not inclusive of those data lost to follow-up (table 2). Interview data confirmed that neither control nor intervention participants found the follow-up outcome assessments particularly burdensome, but some participants reported being unsure of the relevance and purpose of the questions:

Obviously I did them [assessments] because you were doing that and maybe, um, you were looking for something that I can't see. (participant)

I felt quite at ease with the questions and, you know, yes I do or no I don't. (participant)

\section{Objective 5: fidelity of the intervention delivery}

Participants were categorised as having low (attending $<50 \%$ of sessions), medium (attending $50 \%-75 \%$ of sessions), or high (attending $>75 \%$ of sessions) attendance. Of the 20 participants in the intervention arm, $2(10 \%)$ were deemed to be low adherers (neither of whom attended any singing sessions), 4 (20\%) medium adherers and $14(70 \%)$ high adherers.

Facilitators self-reported high levels of adherence to essential elements within the intervention manual, averaging $97 \%$ for one facilitator and $92 \%$ for the other. Video recordings of three intervention sessions (sessions 1, 3 and 8) were sampled for cross-checking of intervention fidelity analysis by a member of the research team. These revealed lower levels of adherence compared with the facilitators' self-reports ( $72 \%$ and $78 \%$, respectively). Although this may indicate some social desirabilityresponding on the part of the facilitators (ie, an exaggeration of their fidelity to the intervention), the overall level of intervention adherence as observed by the research team member can still be considered acceptable. It is also notable that the facilitator-observer discrepancies that were identified were mainly around session elements that were more open to subjective (rater) interpretation (eg, encouragement of participants to support each other).

\section{Objective 6: SPA intervention costs}

Intervention costs were generated for each venue, accounting for different numbers of participants (six or seven participants) and excluding travel costs of participants. The average cost of the intervention per participant based on 2019 unit costs was £399.33 including training costs, £344.56 excluding training costs and $£ 325.62$ excluding training costs and assistant during sessions (which may in some cases be provided by a carer attending the group). The average cost of transport claimed by participant was $£ 89.21$ for attending the sessions. The study also trialled the methods used to collect self-report data on health and social services service utilisation. In the sample here, service use was higher in the intervention compared with the control but the study was not powered to look at these differences. 
Table 1 Baseline demographic characteristics of participants

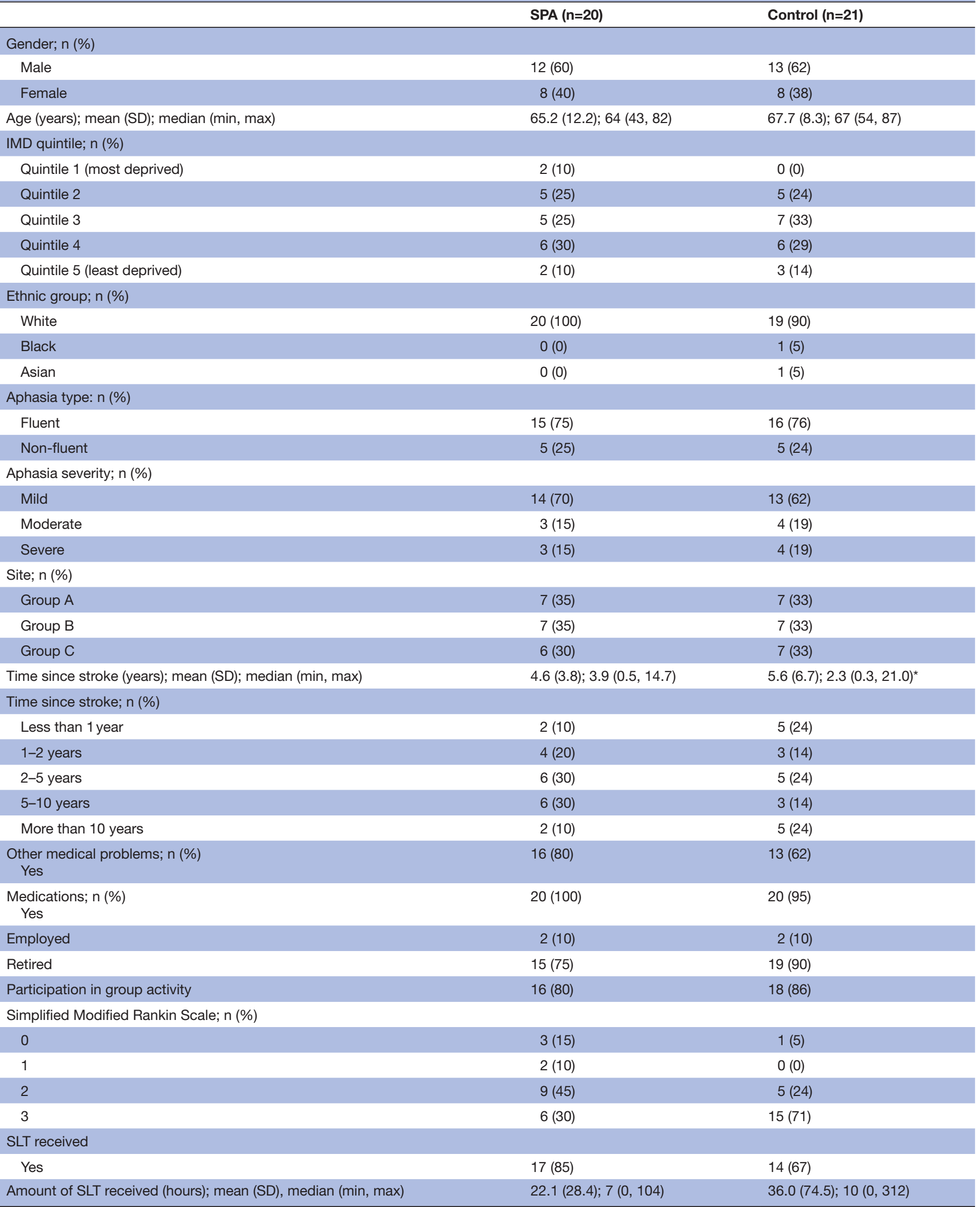

*Based on 20 participants; one extreme outlier excluded. With inclusion of this outlier, mean=8.3 years ( $\mathrm{SD}=4.3)$.

SLT, speech and language therapist; SPA, singing groups for people with aphasia. 
Table 2 Outcome measures at baseline and follow-up assessment points

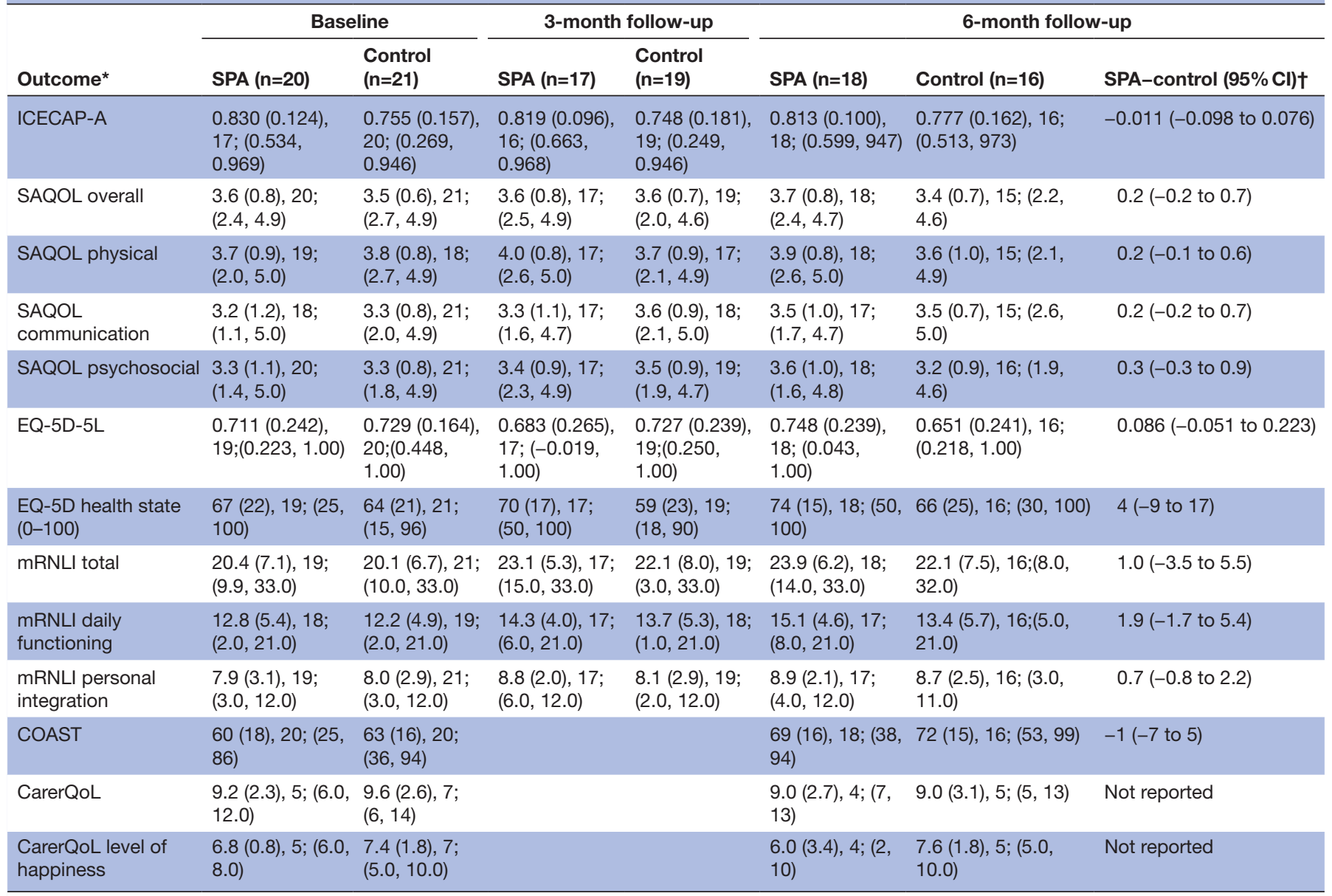

Higher scores indicate better outcomes on outcome measures (ie, greater quality of life, greater reintegration into normal living, more effective communication). *Outcomes are reported as mean (SD), number of cases; (minimum, maximum).

†Analyses adjusted for baseline score, gender, site, aphasia severity and aphasia type.

CarerQoL, carer quality of life; COAST, Communication Outcome after Stroke Scale; EQ-5D-5L, EuroQol Quality of Life Scale ; ICECAP-A, ICEpop CAPability measure for Adults; mRNLI, modified Reintegration to Normal Living Index; SAQOL-39, Stroke and Aphasia Quality of Life Scale; SPA, singing groups for people with aphasia.

Further data on intervention costs and health and social services self-reported use can be found in online supplemental appendix 5 .
Objective 7: trial and intervention acceptability and feasibility for participants, group facilitators and singing champions

Thematic analysis of qualitative interviews identified four overarching themes (online supplemental appendix 6 provides illustrative quotes).

\begin{tabular}{lcll}
\hline \multicolumn{2}{l}{ Table 3} & Sample size estimates for candidate primary outcomes & \\
\hline Outcome measure & MCID & SD & Sample size required \\
\hline EQ-5D* & 0.1 & 0.2 & 284 \\
SAQOL-39† & 0.1 increments from 0.1 to 0.5 & 0.81 & $144-2760$ \\
mRNLIF & 14.8 & 6.8 & 12 \\
ICECAP-A§ & 0.07 & 0.07 & 234 \\
\hline
\end{tabular}

${ }^{*} \mathrm{MCID}$ from Chen et $\mathrm{a}{ }^{51}$; SD from Kind et al. ${ }^{52}$

†MCIDs and SDs from Jones et al..$^{50}$

ҒMCID from https://www.sralab.org/rehabilitation-measures/reintegration-normal-living-index: based on the full version of the RNLI and not the modified version; SD from our own trial sample.

§MCID from Hackert et $\left.a\right|^{53}$; SD from the SPA pilot sample.

EQ-5D, EuroQol Quality of Life Scale ; ICECAP-A, ICEpop CAPability measure for Adults; MCID, minimum clinically important difference; mRNLI, modified

Reintegration to Normal Living Index; SAQOL-39, Stroke and Aphasia Quality of Life Scale; SPA, singing groups for people with aphasia. 
Acceptability of the research process

Participants and carers in both trial arms were positive about the way in which the study had been introduced to them, and mentioned being motivated by an opportunity to do something new and by doing something that may be helpful for others as well as themselves. The facilitators expressed their motivation for working on a research study which combined their interest in music with the possibility of improving the well-being of people with aphasia. Facilitators interacted with the intervention manual in different ways, but overall saw it as a useful source of guidance and information. One facilitator described following the manual throughout, while the other facilitator said that they used it more as a 'guide' while still being their 'own facilitator self'.

Singing champions' motivations for being involved in the study were connected with their own stroke and recovery, as well as their general interest in music. Singing champions were satisfied with their role in the singing programme and the opportunity to participate in research for stroke survivors, and valued the early contact provided with the facilitator. Facilitators valued the singing champions' ability to relate to participants, and appreciated the support that they provided in delivering the sessions.

\section{Perceptions about group development and group bonding}

Participants and singing champions acknowledged the importance of the facilitator's role in fostering a comfortable and supportive environment, while bringing professionalism and expertise to the group sessions. Planned session content was also seen as important in this regard, with aspects such as singing familiar songs, warm-up exercises and breaks to allow for participant interaction (for facilitating group development). Facilitators considered that individual participant characteristics influenced levels of bonding, or cohesion, within the singing group, with one facilitator suggesting that differences along these characteristics could be an obstacle to group bonding. However, one participant's carer, while acknowledging differences (eg, in background and interests) between the group members, did not consider this an issue at all. Indeed, many other interviewees described activities such as group-based goal setting and deciding on a group name as being key to the development of group cohesiveness. Some participants also suggested that practical aspects of the intervention design, including supporting regular attendance, providing adequate space, heating, room set up and even parking and public transport availability, also contributed to group cohesiveness.

\section{Impact of taking part in the SPA intervention}

Participants clearly enjoyed the singing group programme, saying that they felt encouraged by it and that it was something to look forward to each week. Some reported that taking part in the programme had increased their confidence and willingness to try new things, although others suggested that the impact of participation was more fleeting. One participant spoke about a positive change in their aphasia but most participants thought that the group did not impact on their language and communication or reduce the barriers they were facing to joining in with similar activities and groups (eg, in terms of access, time and dependence on others). Facilitators identified several benefits which they attributed to the intervention, starting with the provision of an opportunity for people with aphasia to meet and share experiences with others. In turn, they suggested, this sharing contributed to the development of warm, empathetic friendships.

\section{Aspects of working with people with aphasia}

The facilitators reported that they tailored their 'normal' approach to running singing programmes to meet the needs of people with aphasia. Such modifications were reported in three main categories: session content (eg, offering songs with suitable pitch and tempo), physical considerations (eg, accepting that not everyone can stand up to sing) and general approach (eg, slower approach to support involvement of less able participants). One facilitator felt that they needed more guidance on how to work with participants who have comorbidities. As a result of participants' aphasia, most of the interviews were shorter and the data less complex than may be expected from interviews with participants without these kinds of communication impairment.

\section{Safety}

There were 10 AEs reported across the trial: these were 'real time' AEs reported during the period that the singing programmes were in operation (see table 4). There were five AEs and five serious AEs (SAEs). All events were confined to four participants; one reported five events, one reported three events and two reported one event each. Of the total events reported, seven (three AEs and four SAEs) were unrelated to the trial and included falls, one death and one lung infection. One SAE was possibly related to the trial and involved a fall. The remaining two AEs were related to the trial; one participant fell off a chair and one participant trapped their thumb between chairs. Further information on AEs/SAEs reported at 3-month and 6-month follow-ups can be found in online supplemental appendix 7 .

\section{DISCUSSION}

\section{Summary of main findings}

This RCT piloted a new group-based singing intervention for people with aphasia after stroke, designed to support positive psychosocial outcomes. The pilot RCT met all of its objectives in assessing the acceptability and feasibility of the intervention and trial processes. Recruitment to the trial was acceptable ( $85.41 \%$ of target at randomisation), and randomisation and allocation concealment were both successful, with just two participants revealing their allocation (objective 1). Participant retention rates were also high (83\%; objective 2), and sample size estimates necessary to select outcome measures for a definitive trial 
Table 4 Reports of AEs/ SAEs

\begin{tabular}{|c|c|c|c|c|c|c|c|}
\hline Group & Severity & Total & Related & Probable & Possible & Unrelated & $\begin{array}{l}\text { Reporting events } \\
\text { (n) }\end{array}$ \\
\hline SPA & $A E$ & 4 & $2^{*}$ & 0 & 0 & $2 \dagger$ & 3 \\
\hline \multirow[t]{2}{*}{ Control } & $A E$ & 1 & 0 & 0 & 0 & 1 & 1 \\
\hline & SAE & 0 & 0 & 0 & 0 & 0 & 0 \\
\hline
\end{tabular}

*Events were: fall off chair at singing group; trap thumb between chairs at singing group (two different participants).

†Events were: falls at home (same participant).

fEvent was: fall.

$\S 1 \times$ fall; $1 \times$ death (same participant); $1 \times$ fall, $1 \times$ lung infection (same participant).

$A E$, adverse event; $S A E$, serious $A E$.

were calculated (objective 3). There was a high level of outcome measure completion and participants did not report any issues with completing these (objective 4). The assessment of intervention fidelity indicated good facilitator adherence to the intervention manual (objective $5)$. Average intervention costs per participant were calculated (£353.95 or $£ 360.58$ including opportunity cost of the singing group facilitator attending training; objective $6)$. Finally, the qualitative interviews showed that participants (and some of their carers), facilitators and singing champions found the SPA intervention to be acceptable to them (objective 7). This trial supports earlier research suggesting that the investigation of group-based singing in people with post-stroke aphasia is feasible, ${ }^{23}$ and builds on this with further insights into the design and measurement of group-based singing interventions around psychosocial outcomes.

\section{SPA intervention and trial limitations and optimisation of the ahead of a definitive trial}

Development of the SPA intervention involved the use of prospective optimisation strategies ${ }^{48}$ including focus groups and interviews with key stakeholders, and a test delivery of the planned first session. ${ }^{24}$ The current pilot trial, accompanying qualitative interviews, a post-trial feedback session with facilitators, and researcher reflections constitute additional actions taken to optimise the intervention and trial procedures prior to a definitive trial. The principal changes made to the intervention and trial protocol as a result of the pilot trial are detailed here, along with limitations of the current pilot feasibility RCT.

Recruitment rates in the current trial were lower than predicted, although similar to those achieved in the one other trial of singing interventions for aphasia. ${ }^{23}$ There are likely two main reasons for this. First, a new, independent, singing group programme was introduced in one region which attracted many stroke survivors, making them ineligible for the current trial. Second, some of the SLTs approached during the recruitment phase were reluctant to refer patients to the trial. On questioning, it became clear that these SLTs strongly believed that the intervention would be successful and did not want to deny such assumed benefit to patients who would be randomised to a control group. In preparation for recruitment to a definitive trial, clinicians will be encouraged by the research team to remain in equipoise, thereby helping to ensure that people with aphasia receive full information about the research in order to make an informed decision about their participation.

This pilot was not powered to test the effectiveness of the SPA intervention and so no conclusions can be drawn here about this, or its cost-effectiveness. However, the pilot has allowed for the rehearsal and refinement of key procedures ahead of a definitive trial, including the estimation of sample size requirements for this. Despite requiring a sample size lower than that in the pilot trial (required $\mathrm{N}=12$ ), no differences were detected between the trial arms on the mRNLI. While this may indicate that the intervention did not affect social participation, it could equally reflect the measure's focus on 'reintegration' and people's comfort and freedom in interacting with others, rather than increased participation in broader social settings-an intended psychosocial outcome of the SPA intervention. Thus, the mRNLI may not adequately capture this aspect of social participation. The SAQOL-39 is unsuitable for use in a definitive trial due to the prohibitively large sample size required (upper $\mathrm{N}=2760$ ). In contrast, the samples required for the ICECAP-A (N=234) and EQ-5D-5L (N=284) are both achievable and are therefore the most suitable primary outcome measures for a definitive trial of SPA.

Although not targeted by any of the outcome measures in the current study, the qualitative interviews revealed that participation in the singing programme was experienced by some participants as contributing to an enhanced general sense of social confidence and individual agency. This might suggest value in including a measure which captures this as a secondary outcome in a definitive trial, such as the Stroke Self-Efficacy Questionnaire. ${ }^{49}$ We note that this measure has been used previously with stroke survivors with and without aphasia. ${ }^{4750}$

Finally, researcher observations and discussions with singing facilitators and other stakeholders during the course of the pilot trial identified two minor intervention procedures that will be modified ahead of a definitive 
trial. First, it is possible that researcher presence in this pilot RCT may have constituted additional practical support (eg, welcoming participants to the sessions, preparing refreshments), leaving facilitators more time to focus on group cohesion. A definitive trial would need to minimise researcher presence at group sessions in order to model later implementation in non-trial settings. Accordingly, these practical roles could be assumed by others attending the programme (eg, singing champions, carers, participants) at low cost and, if participants shared such roles, could further encourage interdependence within the group. Second, further guidance for facilitators is planned in order to outline how they can optimise participants' translation into practice of the psychosocial skills developed within the singing group. This will include ideas such as participant action planning (ie, what they intend to do beyond the trial), and using the resource pack to explore new (external) opportunities and celebrating achievements.

\section{CONCLUSIONS}

The SPA intervention targets the psychosocial needs of people with aphasia after a stroke, as these needs are inadequately met by existing services. This is the first RCT of a singing group intervention for people with aphasia designed specifically to address some of these needs. The study indicated that the SPA intervention and trial processes were acceptable to people with aphasia, carers, facilitators and co-facilitator 'singing champions'. Fidelity of intervention delivery was good, with minimal safety concerns, and costs of the intervention delivery were calculated. The study has enabled the prioritisation of outcome measures and provided sample size estimates for these, and has allowed for optimisation of the intervention and trial processes ahead of a planned definitive RCT of SPA.

Twitter Mark Tarrant @MarkTarrant_91, Mary Carter @marydianacarter, Paolo Landa @PaoloneMTL, Chris Code @ChristyMc0de and Ruth A Lamont @ RuthALamont

Acknowledgements The authors express their gratitude to the many individuals that took part in this research. This research would not have been possible without them. We are grateful to our stroke service user group for their support in the development and delivery of this trial. We are also grateful to Sam Abrahams and Anna Batson for supporting delivery of the singing programme, and Anthea Asprey for supporting the qualitative work. We would also like to thank our independent Trial Steering Committee: Professor Stephen Clift, Mrs Barbara Chalk, Mr Justin Smallwood, Dr Doyo Enki and Exeter Clinical Trials Unit.

Contributors MT led the project team and was principal investigator. RC was trial manager and researcher and supported all aspects of the trial. MC was trial researcher, conducted all the interviews and undertook the qualitative analysis. SGD led the process evaluation and supervised the qualitative analysis. RT led the quantitative data analyses and FCW conducted the quantitative data analyses. PL conducted the health economics analysis, supervised by AS. JA was service user group member and supported delivery of the trial, with particular focus on recruitment. CC provided aphasia and speech/language expertise to the research team, including training the singing group facilitators and assessing participant capacity to consent. $A B$ supported trial closure and produced the first draft of the current manuscript. RAL led the completion of the manuscript. All authors commented on and approved the final manuscript. Plymouth Music Zone (PMZ) is an outreach charity that works in partnership with health, community and social care organisations to provide tailored, participatory music services to vulnerable or marginalised groups of all ages across Plymouth. It also enables Music Leaders to work in a diverse range of settings and shares its learning around developing inclusive practice and leadership across the South West of England and further afield. PMZ supported the development and delivery of the SPA intervention. We are grateful to PMZ, and their Creativity and Learning Director Anna Batson in particular, for the support with the SPA project.

Funding The trial is funded by the Stroke Association (QQ12/TSA 2016/14). Excess treatment costs have been covered by South Devon and Torbay Clinical Commissioning Group, North East and West Devon Clinical Commissioning group and the University of Exeter Medical School. This report is independent research supported by the National Institute for Health Research Applied Research Collaboration South West Peninsula.

Disclaimer The views expressed are those of the author(s) and not necessarily those of the NHS, the NIHR or the Department of Health.

Competing interests All authors report the Stroke Association funding for the work under consideration.

Patient consent for publication Not required.

Ethics approval Ethical approval for the study was granted by the Health Research Authority (HRA) and NHS National Research Ethics Service and Research, via the Southwest - Frenchay Research Ethics Committee (17/SW/0060).

Provenance and peer review Not commissioned; externally peer reviewed.

Data availability statement The study data have not been made available. Participants did not consent for datasets to be stored or accessed outside of the research team.

Supplemental material This content has been supplied by the author(s). It has not been vetted by BMJ Publishing Group Limited (BMJ) and may not have been peer-reviewed. Any opinions or recommendations discussed are solely those of the author(s) and are not endorsed by BMJ. BMJ disclaims all liability and responsibility arising from any reliance placed on the content. Where the content includes any translated material, BMJ does not warrant the accuracy and reliability of the translations (including but not limited to local regulations, clinical guidelines, terminology, drug names and drug dosages), and is not responsible for any error and/or omissions arising from translation and adaptation or otherwise.

Open access This is an open access article distributed in accordance with the Creative Commons Attribution Non Commercial (CC BY-NC 4.0) license, which permits others to distribute, remix, adapt, build upon this work non-commercially, and license their derivative works on different terms, provided the original work is properly cited, appropriate credit is given, any changes made indicated, and the use is non-commercial. See: http://creativecommons.org/licenses/by-nc/4.0/.

\section{ORCID iDs}

Mark Tarrant http://orcid.org/0000-0002-7194-1428

Mary Carter http://orcid.org/0000-0002-1063-5814

Sarah Gerard Dean http://orcid.org/0000-0002-3682-5149

Rod Taylor http://orcid.org/0000-0002-3043-6011

Fiona C Warren http://orcid.org/0000-0002-3833-0182

Anne Spencer http://orcid.org/0000-0002-8163-3103

Paolo Landa http://orcid.org/0000-0001-6532-6747

Amy Backhouse http://orcid.org/0000-0001-7356-0525

Ruth A Lamont http://orcid.org/0000-0002-3158-5836

Raff Calitri http://orcid.org/0000-0003-0889-4670

\section{REFERENCES}

1 The Stroke Association. State of the nation stroke: statistics, 2018.

2 Dalemans RJP, De Witte LP, Beurskens AJHM, et al. An investigation into the social participation of stroke survivors with aphasia. Disabil Rehabil 2010;32:1678-85.

3 Northcott S, Moss B, Harrison K, et al. A systematic review of the impact of stroke on social support and social networks: associated factors and patterns of change. Clin Rehabil 2016;30:811-31.

4 Cruice M, Worrall L, Hickson L. Quantifying aphasic people's social lives in the context of non-aphasic peers. Aphasiology 2006;20:1210-25.

5 Vickers CP. Social networks after the onset of aphasia: the impact of aphasia group attendance. Aphasiology 2010;24:902-13.

6 Parr S. Living with severe aphasia: tracking social exclusion. Aphasiology 2007;21:98-123. 
7 Haslam C, Holme A, Haslam SA, et al. Maintaining group memberships: social identity continuity predicts well-being after stroke. Neuropsychol Rehabil 2008;18:671-91.

8 Dworzynski K, Ritchie G, Playford ED. Stroke rehabilitation: longterm rehabilitation after stroke. Clin Med 2015;15:461-4.

9 Le Dorze G, Salois-Bellerose Émilie, Alepins M, et al. A description of the personal and environmental determinants of participation several years post-stroke according to the views of people who have aphasia. Aphasiology 2014;28:421-39.

10 The Stroke Association. Together we can conquer stroke: stroke association strategy 2015 to 2018, 2018.

11 Tallal P, Gaab N. Dynamic auditory processing, musical experience and language development. Trends Neurosci 2006;29:382-90.

12 Patel AD. Why would musical training benefit the neural encoding of speech? the opera hypothesis. Front Psychol 2011;2:142.

13 Zaidel DW. Neuropsychology of art. 2 edn. Hove, East Sussex: Psychology Press, 2016.

14 Cohen NS. The effect of singing instruction on the speech production of neurologically impaired persons. J Music Ther 1992;29:87-102.

15 Hurkmans J, de Bruijn M, Boonstra AM, et al. Music in the treatment of neurological language and speech disorders: a systematic review. Aphasiology 2012;26:1-19.

16 Schlaug G, Norton A, Marchina S, et al. From singing to speaking: facilitating recovery from nonfluent aphasia. Future Neurol 2010;5:657-65.

17 Tomaino CM. Recovery of fluent speech through a musician's use of pre-learned song repertoire: a case study. Music Med 2010;2:85-8.

18 Monroe P, Halaki M, Kumfor F, et al. The effects of choral singing on communication impairments in acquired brain injury: a systematic review. Int J Lang Commun Disord 2020;55:303-19.

19 Lanyon L, Worrall L, Rose M. What really matters to people with aphasia when it comes to group work? A qualitative investigation of factors impacting participation and integration. Int J Lang Commun Disord 2018:53:526-41.

20 Haslam SA, Jetten J, Postmes T, et al. Social identity, health and Well-Being: an emerging agenda for applied psychology. Appl Psychol 2009;58:1-23.

21 Tamplin J, Baker FA, Jones B, et al. 'Stroke a CHORD': the effect of singing in a community choir on mood and social engagement for people living with aphasia following a stroke. NeuroRehabilitation 2013;32:929-41.

22 Mantie-Kozlowski A, Mantie R, Keller CH. Enjoyment in a recreationa sing-along group for people with aphasia and their caregivers. Aphasiology 2018;32:518-37.

23 Zumbansen A, Peretz I, Anglade C, et al. Effect of choir activity in the rehabilitation of aphasia: a blind, randomised, controlled pilot study. Aphasiology 2017;31:879-900.

24 Tarrant M, Warmoth K, Code C, et al. Creating psychological connections between intervention recipients: development and focus group evaluation of a group singing session for people with aphasia. BMJ Open 2016;6:e009652.

25 Craig P, Dieppe P, Macintyre S, et al. Developing and evaluating complex interventions: the new medical Research Council guidance. BMJ 2008;337:a1655

26 Fisher WA, Fisher JD, Harman J. The information-motivationbehavioral skills model: a general social psychological approach to understanding and promoting health behavior. social psychological foundations of health and illness. Malden: Blackwell Publishing, 2003: 82-106.

27 Tarrant M, Carter M, Dean SG, et al. Singing for people with aphasia (spa): a protocol for a pilot randomised controlled trial of a group singing intervention to improve well-being. BMJ Open 2018;8:e025167.

28 Eldridge SM, Chan CL, Campbell MJ, et al. Consort 2010 statement: extension to randomised pilot and feasibility trials. BMJ 2016;355:i5239.

29 Borek AJ, Abraham C, Smith JR, et al. A checklist to improve reporting of group-based behaviour-change interventions. BMC Public Health 2015;15:963.
30 O'Brien BC, Harris IB, Beckman TJ, et al. Standards for reporting qualitative research: a synthesis of recommendations. Acad Med 2014;89:1245-51.

31 National Institute for Health Research. Engaging with people who have aphasia: a set of resources for stroke researchers. NIHR CRN, 2014.

32 Stroke Association. Accessible information guidelines: making information accessible for people with aphasia, 2012.

33 Al-Janabi H, Flynn TN, Coast J. Development of a self-report measure of capability wellbeing for adults: the ICECAP-A. Qual Life Res 2012;21:167-76.

34 Hilari K, Byng S, Lamping DL, et al. Stroke and aphasia quality of life Scale-39 (SAQOL-39). Stroke 2003;34:1944-50.

35 Herdman M, Gudex C, Lloyd A, et al. Development and preliminary testing of the new five-level version of EQ-5D (EQ-5D-5L). Qual Life Res 2011;20:1727-36.

36 Miller A, Clemson L, Lannin N. Measurement properties of a modified reintegration to normal living index in a communitydwelling adult rehabilitation population. Disabil Rehabil 2011;33:1968-78.

37 Long A, Hesketh A, Paszek G, et al. Development of a reliable self-report outcome measure for pragmatic trials of communication therapy following stroke: the communication outcome after stroke (coast) scale. Clin Rehabil 2008;22:1083-94.

38 Powell GE, Bailey S, Clark E. A very short version of the Minnesota aphasia test. Br J Soc Clin Psychol 1980;19:189-94.

39 Brouwer WBF, van Exel NJA, van Gorp B, et al. The CarerQol instrument: a new instrument to measure care-related quality of life of informal caregivers for use in economic evaluations. Qual Life Res 2006;15:1005-21.

40 Craig LE, Wu O, Bernhardt J, et al. Approaches to economic evaluations of stroke rehabilitation. Int J Stroke 2014;9:88-100.

41 Powell GE, Clark E, Bailey S. Categories of aphasia: a cluster-analysis of Schuell test profiles. Br J Disord Commun 1979;14:111-22.

42 Baker C, Worrall L, Rose M, et al. 'It was really dark': the experiences and preferences of people with aphasia to manage mood changes and depression. Aphasiology 2020;34:19-46.

43 Sekine K, Rose ML, Foster AM, et al. Gesture production patterns in aphasic discourse: in-depth description and preliminary predictions. Aphasiology 2013;27:1031-49.

44 Browne RH. On the use of a pilot sample for sample size determination. Stat Med 1995;14:1933-40.

45 Curtis LA, Burns A. Unit costs of health and social care. Personal Social Services Research Unit, University of Kent, 2019.

46 Braun V, Clarke V. Using thematic analysis in psychology. Qual Res Psychol 2006;3:77-101.

47 Dean SG, Poltawski L, Forster A, et al. Community-based rehabilitation training after stroke: results of a pilot randomised controlled trial (retrain) investigating acceptability and feasibility. BMJ Open 2018;8:e018409.

48 Levati S, Campbell P, Frost R, et al. Optimisation of complex health interventions prior to a randomised controlled trial: a scoping review of strategies used. Pilot Feasibility Stud 2016;2:17.

49 Jones F, Partridge C, Reid F. The stroke self-efficacy questionnaire: measuring individual confidence in functional performance after stroke. J Clin Nurs 2008;17:244-52.

50 Jones $\mathrm{F}$, Gage H, Drummond A, et al. Feasibility study of an integrated stroke self-management programme: a clusterrandomised controlled trial. BMJ Open 2016:6:e008900.

51 Chen P, Lin K-C, Liing R-J, et al. Validity, responsiveness, and minimal clinically important difference of EQ-5D-5L in stroke patients undergoing rehabilitation. Qual Life Res 2016;25:1585-96.

52 Kind P, Hardman G, Macran S. UK population norms for EQ-5D. Centre for Health Economics, University of York, 1999.

53 Hackert MQN, van Exel J, Brouwer WBF. Does the ICECAP-O cover the physical, mental and social functioning of older people in the UK? Qual Life Res 2019;28:761-70. 